The Journal of Laryngology \& Otology

http://journals.cambridge.org/JLO

Additional services for The Journal of Laryngology \& Otology:

Email alerts: $\underline{\text { Click here }}$

Subscriptions: $\underline{\text { Click here }}$

Commercial reprints: $\underline{\text { Click here }}$

Terms of use : $\underline{\text { Click here }}$

\title{
Validation of sonotubometry in healthy adults
}

S J C van der Avoort, N van Heerbeek, G A Zielhuis and C W R J Cremers

The Journal of Laryngology \& Otology / Volume 120 / Issue 10 / October 2006, pp 853 - 856

DOI: 10.1017/S0022215106001095, Published online: 31 March 2006

Link to this article: http://journals.cambridge.org/abstract_S0022215106001095

How to cite this article:

S J C van der Avoort, N van Heerbeek, G A Zielhuis and C W R J Cremers (2006). Validation of sonotubometry in healthy adults. The Journal of Laryngology \& Otology, 120, pp 853-856 doi:10.1017/S0022215106001095

Request Permissions : $\underline{\text { Click here }}$ 


\title{
Validation of sonotubometry in healthy adults
}

\author{
S J C van der Avoort, MD, N van Heerbeek, MD, PhD, G A Zielhuis, PhD*, \\ C W R J CREMERs, MD, PhD
}

\begin{abstract}
Background: Frequent active opening of the eustachian tube (ET) allows ventilation of the middle ear and equilibration of pressure changes. Active opening is accomplished by the contraction of the paratubal muscles during swallowing. Because a disturbance of the ventilatory function of the ET may contribute to the development of otitis media with effusion, it is important to investigate ET function. Sonotubometry can be used to detect whether the ET can open or not during swallowing acts.

Methods: We developed a sonotubometer to test ET ventilatory function in 36 healthy adults. The width of the test signal frequency was between 5500 and $8500 \mathrm{~Hz}$ (centre frequency of $7000 \mathrm{~Hz}$ ) and the loudness was $95 \mathrm{~dB}$. To test reproducibility, testing took place in two sessions of 10 swallowing acts each.

Results: Opening of the ET could be registered in 91.6 per cent of the subjects in at least one of the two measurements. The first and the second measurements were highly correlated, with a Spearman's coefficient of 0.907 .

Conclusion: We confirmed that there is generally a good ventilatory ET function in otologically healthy adults, although, in a few cases, ET opening was not registered. Furthermore, we confirmed that our sonometric test equipment had acceptable reproducibility. Sonotubometry is a promising method for assessing ventilatory ET function. Research is ongoing to test the discriminative power of sonotubometry in children with various otological conditions.
\end{abstract}

Key words: Eustachian Tube; Sound; Ventilation; Physiology; Sonotubometry

\section{Introduction}

The most important functions of the eustachian tube (ET) are ventilation, protection and clearance of the middle ear. ${ }^{1,2}$ Gases are exchanged continuously between the tympanic cavity and the middle-ear mucosa via diffusion. Regular, active opening of the ET allows ventilation of the middle ear and equilibration of pressure changes. Active opening is accomplished by the contraction of the paratubal muscles. These muscles contract during swallowing, yawning or movement of the mandible. However, the ET does not open during each swallow.

Because disturbance of any of the ET functions may contribute to the development of otitis media with effusion, ${ }^{3-11}$ it is important to investigate ET function. Investigations of ET function have mainly measured its ventilatory aspect. In addition to manometric function tests ${ }^{12-16}$ and indirect tubal function tests (e.g. tympanometry, pure tone audiometry and otoscopy), sonotubometry ${ }^{17-27}$ can be used to detect whether the ET can open or not. The latter function test has some advantages compared with the former tests. ${ }^{28}$ Sonotubometry takes place under physiological circumstances, can be performed on ears with an intact tympanic membrane (in contrast to manometric tests) and is easily tolerated by the subject. Sonotubometry is based on the principle that sound that is applied to the nasopharyngeal ostium of the ET is conducted through the ET to the middle ear. During active opening of the ET, more sound will be recorded in the external auditory canal. Several investigators have studied sonotubometry to determine its usefulness in assessing ET ventilatory function. ${ }^{17-27}$

However, thus far, this test has still not been applied in clinical practice, partly because there is currently no consensus on test equipment and test properties in the literature, and partly because the test's reproducibility and clinical value are yet to be demonstrated. It is therefore argued that sonotubometry needs further improvement. Although various reports have been published on sonotubometry in adults, it appears to be difficult to obtain reliable, positive test results in more than 60 per cent of healthy ears. 
Moreover, the question of the test's reproducibility remains.

The aim of this study was to improve the performance of sonotubometry in adults.

\section{Methods and materials}

The equipment used in the present investigation is schematically illustrated in Figure 1.

A test signal was produced by a computer using Cool Edit Pro 2.0 software (Syntrillium Software Corporation, USA) and delivered to the nasopharyngeal ostium of the ET using an Ear Tone 3A insert phone (Auditory Systems, Indianapolis, USA) fixed into one of the nostrils of the subject with a foam ear tip (this tip adjusted itself to the shape of the nostril to prevent leakage of sound). A probe microphone (Etymotic Research-7c, Illinois, USA) was placed in the ipsilateral external auditory canal and also fixed with a foam ear tip to prevent loss of test sound and interference by background noises. The microphone was connected to the computer and the sound recorded using the Cool Edit Pro 2.0 software.

Earlier studies on sonotubometry ${ }^{17-27}$ were considered carefully in order to choose the optimal test properties. We started with a high frequency test sound, following the work of Virtanen, who showed that only frequencies higher than $5000 \mathrm{~Hz}$ were appropriate. ${ }^{17}$ A pure tone test signal was used with a frequency of $7000 \mathrm{~Hz}$ and an initial loudness of $75 \mathrm{~dB}$. Using these settings, we could register tubal openings in 60 per cent of a population of healthy adults. However, this result was disappointing compared with earlier publications, so our equipment or test properties needed improvement. Thus, loudness was increased to $95 \mathrm{~dB}$ (as the test signal might have been too low to be detected by the probe microphone in the external ear canal).

The use of a pure tone signal was also considered a reason for the false negative test responses. In a unilateral closed tube, e.g. the external ear canal, a pure tone sound can cause a standing wave pattern. A standing wave pattern consists of nodes and antinodes. Measurement in the centre of a node can generate false negative results. So, to prevent the formation of standing wave patterns, the test signal was changed from a pure tone signal to a band signal

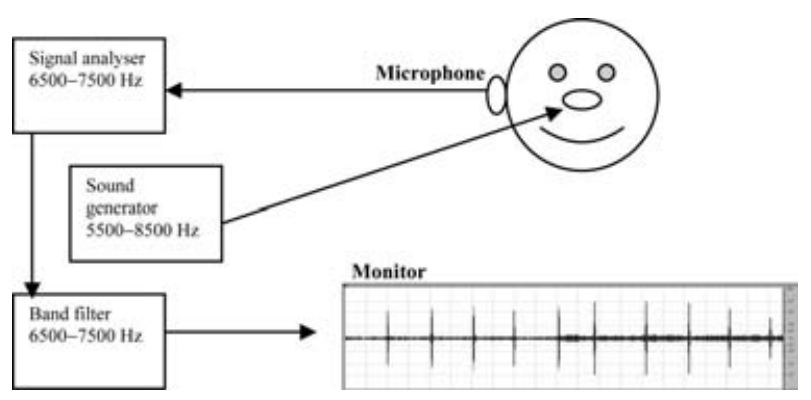

FIG. 1

Diagram of equipment used. between 5500 and $8500 \mathrm{~Hz}$, with a centre frequency of $7000 \mathrm{~Hz}$.

We thus chose to use a sound with a bandwidth between 5500 and $8500 \mathrm{~Hz}$ and a fixed loudness of $95 \mathrm{~dB}$. The sound level in the external ear canal was continuously measured and these data stored. Off-line, the recordings were band pass filtered with a central frequency of $7000 \mathrm{~Hz}$, with a minimum of $6500 \mathrm{~Hz}$ and a maximum of $7500 \mathrm{~Hz}$. Filtering was applied to minimize ambient noise and sounds due

(a)

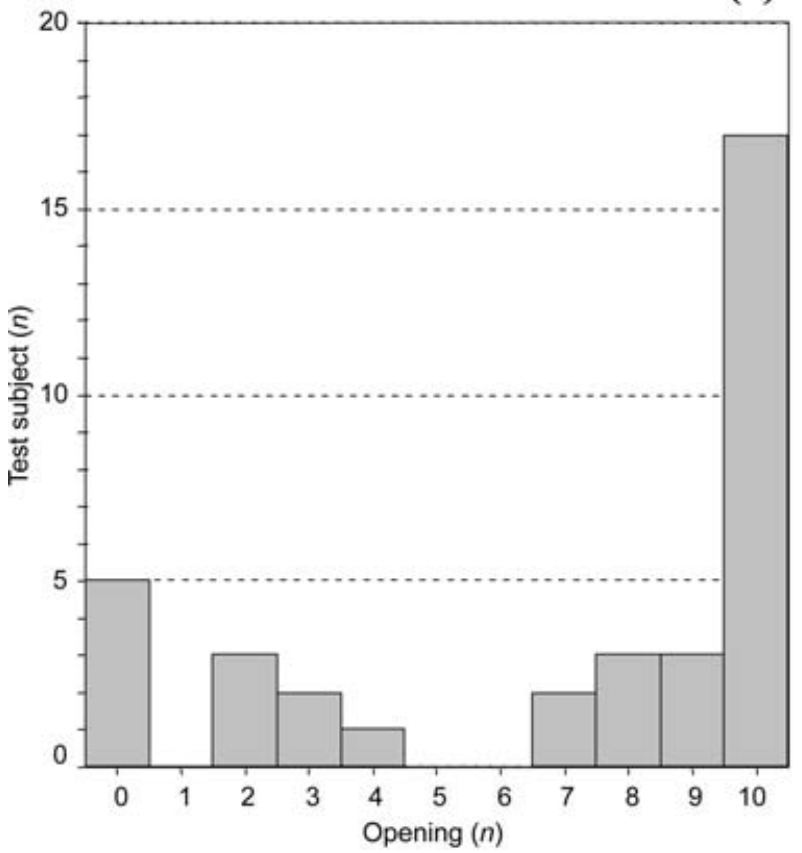

(b)

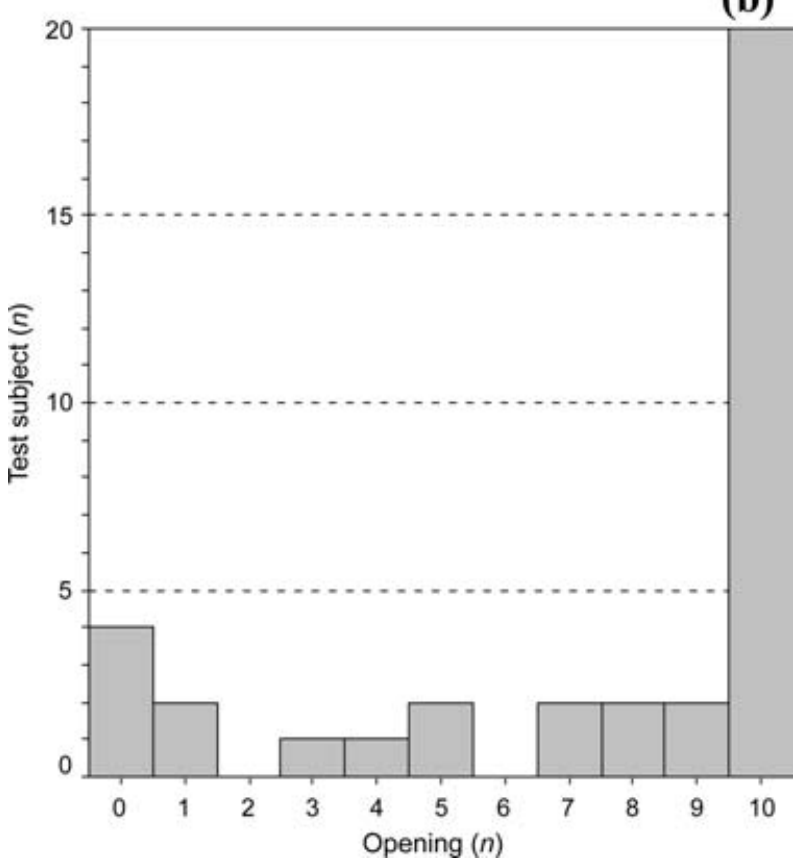

FIG. 2

Eustachian tube openings in the (a) first and (b) second test measurements ( $n=36$ subjects). 
to swallowing. Discrimination between a positive and a negative response was done by visual evaluation. Furthermore, a measured peak was only counted as a positive response if it occurred simultaneously with a swallowing act.

To evaluate the patency of the ET, subjects were instructed to swallow water 10 times, in a sitting position, at 10 -second intervals. After 10 swallows, the microphone and the sound source were removed and, after a short 5-minute break, replaced for the second measurement. The second measurement was performed in an identical fashion to the first measurement in order to test its reproducibility.

The test population consisted of 36 adults, 12 men and 24 women. Their ages varied from 18 to 55 years, with a mean age of 33 years. Only otologically healthy subjects were included; individuals with a history of middle-ear surgery, recurrent otitis media or complaints of tubal dysfunction were excluded.

\section{Results}

Figure 2 shows the results of the sonotubometric measurements. At least one opening of the ET was registered in 31 of the 36 ears in the first measurement and in 32 of the 36 ears in the second measurement. Combining the results of the two measurements per subject, ET opening could be registered in at least one of the measurements in 33 of the 36 tested ears (91.6 per cent). In the other three cases, not a single ET opening was detected. The number of ET openings varied from zero to 10 out of 10 swallowing acts. Figure 3 shows the reproducibility of the test. In 23 of the 36 ears (64 per cent), there was no difference in number of openings between the first and the second measurements. The difference in the number of openings in the

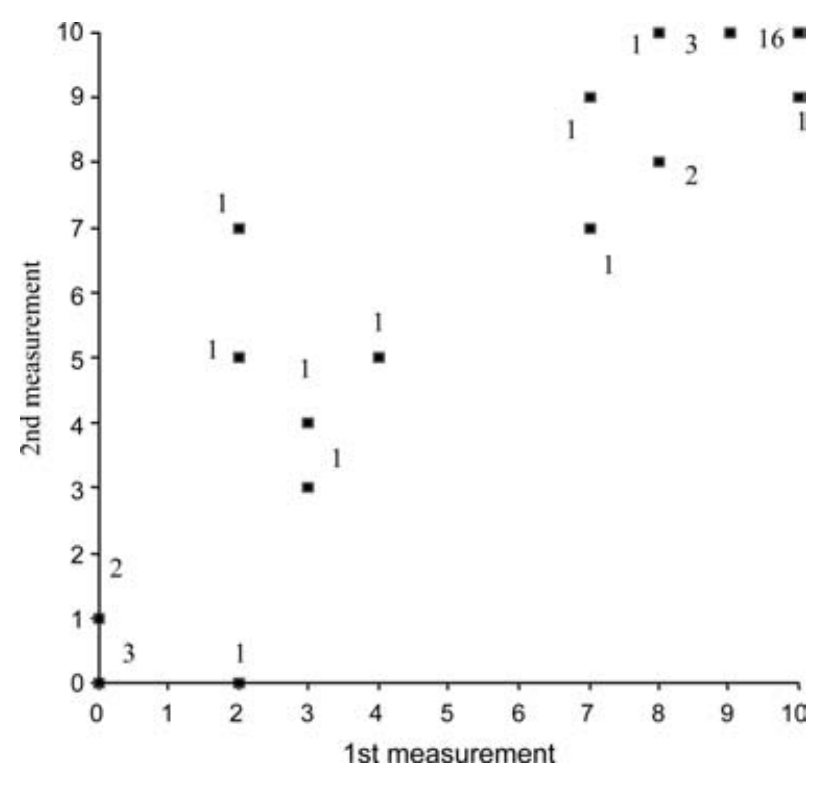

FIG. 3

Reproducibility of eustachian tube opening assessment, comparing the first and second test measurement results. One square represents one or more test persons. first test compared with those in the second test was one in eight subjects ( 22 per cent) and two or more in five subjects (14 per cent), with a maximum difference of five. The first and second measurements were highly correlated, with a Spearman's correlation coefficient of $0.91(p<0.001)$.

\section{Discussion}

The purpose of this study was (a) to develop a sonotubometer, and (b) to test whether sonotubometry was a valid and reproducible method of measuring ET ventilatory function. A non-invasive, physiological method of testing ET ventilatory function is not currently available in ENT practice. Previous studies investigated the role of sonotubometry in the assessment of ET function, but, despite promising results, insufficient confirmation existed to introduce sonotubometry as a routine tubal test in ENT practice. $^{28}$

- Sonotubometry is based on the principle that sound applied to the nasopharyngeal ostium of the eustachian tube (ET) is conducted through the ET to the middle ear; during active opening of the ET, more sound will be recorded in the external auditory canal

- This report describes a sonotubometer developed to test ET ventilatory function in $\mathbf{3 6}$ healthy adults. The width of the test signal frequency was between 5500 and $8500 \mathrm{~Hz}$ (centre frequency of $7000 \mathrm{~Hz}$ ) and loudness was $95 \mathrm{~dB}$

- Sonotubometry is a promising method of assessing ventilatory ET function. Research is ongoing to test the discriminative power of sonotubometry in children with otological conditions

Earlier studies ${ }^{17-27}$ were considered thoroughly when developing the test equipment used in this study. As described in the methods, we initially used a high frequency test sound, following the work of Virtanen. ${ }^{17}$ Later, the loudness used was increased to $95 \mathrm{~dB}$. To prevent the formation of standing wave patterns, the test signal was changed from a pure tone signal to a band signal of between 5500 and $8500 \mathrm{~Hz}$ with a centre frequency of $7000 \mathrm{~Hz}$. With these properties, the testing was performed under the same conditions as the first measurements. Thirty-six healthy adults were tested, and ET openings could be registered in 33 subjects (91.6 per cent). This response is similar to the highest positive response found in earlier studies. ${ }^{17,21}$ It indicates that, in a otologically healthy adult population, there is generally good ET ventilatory function; however, in some cases, no ET openings were registered during 20 swallowing acts. These subjects had no complaints of ET dysfunction, and otoscopy did not show any ET disturbances. Thus, a much lower incidence of ET opening during swallowing acts is probably needed to 
ensure sufficient ET function. Also, ET dysfunction may not always lead to middle-ear problems.

Furthermore, we confirmed that our sonotubometry test method had acceptable reproducibility (i.e. negligible test-retest variability).

As there is currently no physiological ET function test applicable to ears with an intact tympanic membrane and usable in daily ENT practice, these results are promising. Additional research is needed to evaluate the role of sonotubometry as a tubal function test. Such studies in children with various otological conditions are needed to substantiate the benefits of sonotubometry for routine ENT consultations.

\section{Acknowledgment}

The authors thank Ad Snik for his help with finetuning the test equipment and reviewing the manuscript.

\section{References}

1 Bluestone CD, Paradise JL, Beery QC. Physiology of the Eustachian tube in the pathogenesis and management of middle ear effusions. Laryngoscope 1972;82(9):1654-70

2 Bluestone CD. Pathogenesis of otitis media; role of the Eustachian tube. Pediatr Infect Dis J 1996;15:281-91

3 Takahaski M, Takahaski H, Sato H, Honjo I. Primary deficits in the Eustachian tube function in patients with otitis media with effusion. Arch Otolaryngol Head Neck Surg 1989;115:581-4

4 Stenstrom C, Bylander Groth A, Ingvarsson L. Eustachian tube function in otitis prone and healthy children. Int $J$ Pediatr Otorhinolaryngol 1991:21:127-38

5 Bylander Groth A, Stenstrom C. Eustachian tube function and otitis media in children. Ear Nose Throat J 1998;77: $762-9$

6 Bluestone CD, Paradise JL, Beery QC. Physiology of the Eustachian tube in the pathogenesis and management of middle ear effusions. Laryngoscope 1972;82(9):1654-70

7 Sadé J. Pathology and pathogenesis of secretory otitis media. Arch Otolaryngol 1966;84:297-305

8 Sadé J. The nasopharynx, eustachian tube and otitis media. J Laryngol Otol 1994;108:95-100

9 Maw RA. Glue Ear in Childhood. Cambridge: MacKeit Press \& Cambridge University Press, 1995

10 Virtanen H, Palva T. The patulous Eustachian tube and chronic middle ear disease. Acta Otolaryngol 1982; 93(1-2):49-53

11 Sadé J, Ar A. Middle ear and auditory tube: middle ear clearance, gas exchange and pressure regulation. Otolaryngol Head Neck Surg 1997;116:499-524

12 Van Heerbeek N, Ingels KJ, Snik AF, Zielhuis GA. Reliability of manometric Eustachian tube function tests in children. Otol Neurotol 2001;22:183-7

13 Bylander A, Tjernstrom O, Ivarsson A. Pressure opening and closing functions of the Eustachian tube in children and adults with normal ears. Acta Otolaryngol 1983;95: $55-62$

14 Falk B, Magnuson B. Test-retest variability of the Eustachian tube responses in children with persistent middle ear effusion. Arch Otorhinolaryngol 1984;240:145-52
15 Groth P, Ivarsson A, Tjernstrom O. Reliability in tests of the Eustachian tube function. Acta Laryngol 1982;93: 261-7

16 Bluestone CD, Cantekin EI. Current clinical methods, indications and interpretation of Eustachian tube function tests. Ann Otol Rhinol Laryngol 1981;90(6 Pt 1):552-62

17 Virtanen H. Sonotubometry, an acoustical method for objective measurement of auditory tubal opening. Acta Otolaryngol 1978;86:93-103

18 Holmquist J, Björkman G, Olén L. Measurement of Eustachian tube function using sonotubometry [in English]. Scand Audiol 1981;10:33-5

19 Jonathan DA, Chalmers P, Wong K. Comparison of sonotubometry with tympanometry to assess Eustachian tube function in adults. Br J Audiol 1986;20:231-5

20 Jonathan DA. Sonotubometry: its role in childhood glue ear. Clin Otolaryngol 1989;14:151-4

21 Okubo J, Watanabe I, Shibusawa M, Ishikawa N, Ishida H, Teramura K. Sonotubometric measurement of the Eustachian tube function by means of band noise; a clinical view of the acoustic measurement of the Eustachian tube. ORL J Otorhinolaryngol Relat Spec 1987;49:242-5

22 Palva T, Marttila T, Jauhiainen T. Comparison of pure tones and noise stimuli in sonotubometry. Acta Otolaryngol (Stockh) 1987;103:212-16

23 Jonathan DA. The predictive value of eustachian tube function (measured with sonotubometry) in the successful outcome of myringoplasty. Clin Otolaryngol 1990;15: 431-4

24 McBride T, Cunningham M, Derkay C, Doyle W. Evaluation of non-invasive Eustachian tube function tests in normal adults. Laryngoscope 1988;98:655-8

25 Virtanen H. Relation of the body posture to the Eustachian tube function. Acta Otolaryngol 1983;95(1-2):63-7

26 Leider J, Hamlet S, Schwan S. The effect of swallowing bolus and head position on Eustachian tube function via sonotubometry. Otolaryngol Head Neck Surg 1993;109: $66-70$

27 Iwano T, Ushiro K, Yukawa N, Doi T, Kinoshita T, Hamada E et al. Active opening function of the human Eustachian tube: comparison between sonotubometry and pressure equilibration test. Acta Otolaryngol Suppl 1993;500:62-5

28 Van der Avoort S, Van Heerbeek N, Zielhuis G, Cremers C. Sonotubometry - Eustachian tube ventilatory function test; a review. Otol Neurotol 2005;26:538-43

Address for correspondence:

Dr Stijn J C van der Avoort, MD,

Dept of Otorhinolaryngology,

Radboud University Nijmegen Medical Centre,

Philips van Leydenlaan 15,

PO Box 9101,

6500 HB Nijmegen,

The Netherlands.

Fax: + 31243540251

E-mail: s.vanderavoort@kno.umcn.nl

Dr S van der Avoort takes responsibility for the integrity of the content of the paper.

Competing interests: None declared 